\title{
Performance of Correlation Filters in Facial Recognition
}

\author{
Everardo Santiago-Ramirez, J.A. Gonzalez-Fraga, and J.I. Ascencio-Lopez \\ Facultad de Ciencias, Universidad Autónoma de Baja California, Km. 103, Carretera Tijuana- \\ Ensenada, Ensenada, Baja California C. P. 22860 \\ \{everardo.santiagoramirez, angel_fraga, ascencio\}@uabc.edu.mx
}

\begin{abstract}
In this paper, we compare the performance of three composite correlation filters in facial recognition problem. We used the ORL (Olivetti Research Laboratory) facial image database to evaluate K-Law, MACE and ASEF filters performance. Simulations results demonstrate that K-Law nonlinear composite filters evidence the best performance in terms of recognition rate (RR) and, false acceptation rate (FAR). As a result, we observe that correlation filters are able to work well even when the facial image contains distortions such as rotation, partial occlusion and different illumination conditions.
\end{abstract}

Keywords: Facial Recognition, Correlation Filters, PSR performance.

\section{Introduction}

The facial biometric recognition is an important tool for the nonintrusive identification of a person. Although, this is a challengeable task because of the facial variability caused over the time, such as age sign, facial marks, beard, mustache, occlusion, and others, as to changing dress-looking wearing glasses, sunglasses, hats, scarves, and the variation of physical positions. All these problems must be taken into account in personal appearance that a face may suffer due to the different sources and facial expressions.

Biometric recognition algorithms try to match a biometric feature with a template stored in a database [1]. Thus, correlation filters are excellent candidates for the precision of matching in presence of geometric variability and tolerance to noise presented in facial images. Currently, there are few proposals that make use of correlation filters in face recognition problem, which opens a great field of research for the development of robust and efficient algorithms for face recognition.

Some advantages of the correlation filters are: a) they can use all the information of the object, i.e., both form and content (color and intensity) [2][3], b) has a good mathematical foundation [4], c) its design may include geometric distortion invariance and tolerance to certain types of noise (additive, background, light, etc.) and, d) are good candidates to be implemented with fast algorithms as the Fast Fourier Transform (FFT). 
Based on these characteristics, this work presents the performances for three correlation filters: a) K-Law nonlinear composite filter, b) minimum average of correlation energy (MACE) filter and, c) average of synthetic exact filters (ASEF). The rest of paper is organized as follow. Section 2 presents the mathematical foundation of the correlation filters evaluated. Section 3 presents and discusses the performances of the Correlation Filters in Face Recognition. Finally, section 4 presents the conclusions of this work.

\section{Correlation Filters}

Correlation is a robust technique in pattern recognition and is used in many computer applications, such as target automatic recognition, biometrics recognition, phoneme recognition, optical character recognition [2], etc.

The correlation in the frequency domain, as show in Fig. 1, is performed by applying the fast Fourier transform (FFT) over a composite filter (synthesized with the training images set) and a test image, an element-wise multiplication of the FFT of the test image and the filter and, the inverse of fast Fourier transform (IFFT) of this product yield the correlation output.

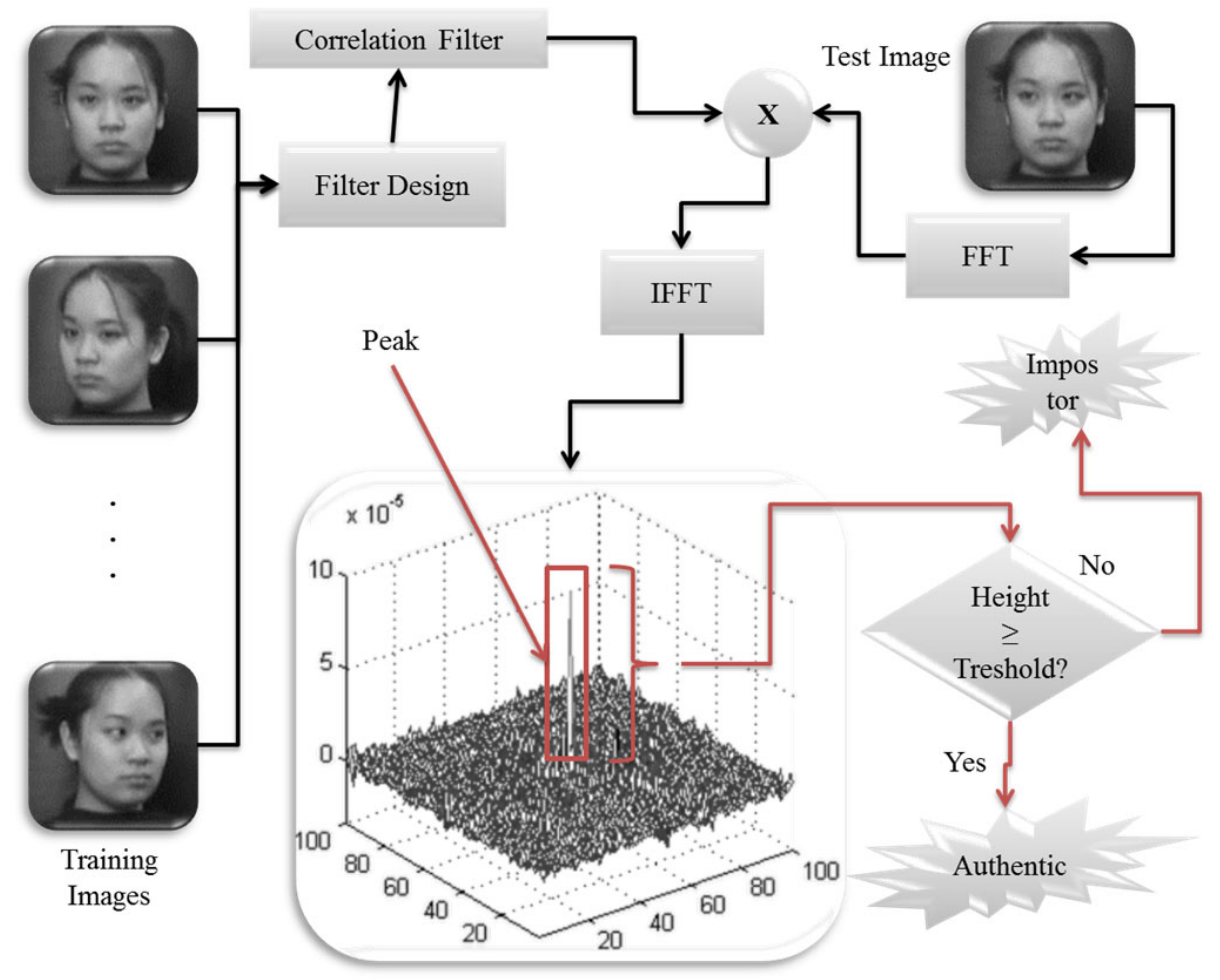

Fig. 1. Facial recognition process by composite correlation filters 
A well-designed filter produces a sharp correlation peak for the true class objects (targets known). To determine a matching between a biometric template and a test facial image, it is necessary to measure the sharpness correlation peak. A good measure for the peak sharpness is the peak-to-sidelobe ratio (PSR), which is presented in equation 1 . In this work, PSR is obtained from a $11 \times 11$ window centered at the correlation peak.

$$
P S R=\frac{(\text { peak }- \text { mean of side lobe region })}{\text { Standard desviation of side lobe region }}
$$

\subsection{K-Law Nonlinear Composite Filter}

Let $x_{1}(x, y), x_{2}(x, y), \ldots, x_{N}(x, y)$ as the $N$ training images with $d$ pixels each one. We rearrange each image as a column vector by lexicographic ordering, i.e., from left to right and top to bottom. This operation produces a vector with $d$ elements. Let $X$ be a matrix of dimension $d$ rows $\times N$ columns, where each column is a training mage. Thus the expression for a basic composite SDF filter is [5]:

$$
h=X\left(X^{+} X\right)^{-1} u,
$$

where + is the transpose conjugate, -1 is the inverse of the matrix and $u$ is a vector that contains correlation values desired for each image in the training set. Generally, the vector $u$ is assigned values of 1 for true class objects, while 0 is assigned to the false class objects.

To improve the performance of composite filters in terms of discrimination against objects similar to the target, correlation-peak sharpness, and correlation noise robustness we apply nonlinear filtering techniques to composite filters such as in reference [6].

In order to apply the nonlinearity on Fourier domain to the filter in equation 2, let $\tilde{X}$ a matrix which contains in column vector form all Fourier transforms of the training images. When a non-linearity is applied to the matrix, the nonlinear operation is applied on each element of the matrix. Hence, the K-Law nonlinearity applied to the matrix $\tilde{X}$ can be described as:

$$
\tilde{X}^{k}=|\tilde{X}|^{k} \exp \left[i \varphi_{\tilde{X}}\right], 0 \leq k \leq 1,
$$

where $|\tilde{X}|$ is the modulus of $\tilde{X}$ and $\varphi$ is its phase. The $k$ value controls the strength of the non-linearity. Now, modifying the equation 2 for build a K-Law SDF composite filter is obtained [6]

$$
\widetilde{H}^{k}=\tilde{X}^{k}\left(\tilde{X}^{k+} \tilde{X}^{k}\right)^{-1} u
$$

When the filter is synthesized with only one image and by setting $k=1$ we get a classical matched filter and when $k=0$, establishing the magnitude of all frequencies to 1 , thus we get a phase-only filter,

The nonlinear operator raises to the $k$-th power the magnitude of the Fourier spectrum of both the analyzed image and the filter, while keeping intact the phase information. This characteristic cause that the filter have a good discrimination capacity. Based on many experiments we determined that the nonlinearity factor $k=0.3$ offers the best performance for k-law filter in facial recognition. 
The K-Law filters, using appropriates images, may be scale and rotation invariant, distortions presented for test images. Also, they have greater tolerance to additive noise. Also, this filter yields a correlation plane with a sharp and strength peak while that a conventional SDF filter doesn't. For this reasons, we chose the filter K-Law for to evaluate his performance in facial recognition problem.

\subsection{Minimum Average Correlation Energy}

The MACE filter was developed for minimize large side-lobes produced by the SDF filters and is represented in equation (5) [2]:

$$
\mathrm{h}=\mathrm{D}^{-1} \mathrm{X}\left(\mathrm{X}^{+} \mathrm{D}^{-1} \mathrm{X}\right)^{-1} \mathrm{u}
$$

Let a training set $\left\{x_{1}, x_{2}, x_{3}, \ldots, x_{N}\right\}$ containing $\mathrm{N}$ images with $d$ pixels each one, then the average energy over all training images is the diagonal matrix $D=$ $\frac{1}{d . N} \sum_{i=1}^{N} \tilde{x}_{i} \tilde{x}_{i}^{*}($ size $d \times d) . \tilde{x}_{i}$ is the FT of each training image and $*$ represents the complex conjugate.

\subsection{Average of Synthetic Exact Filters}

For the ASEF filter, each training image $x_{i}$ is associated to a desired output $y_{i}$, the desired output $y_{i}$ is an output plane of correlation with the peak center over the object of interest. For each pair $\mathrm{x}_{\mathrm{i}}, \mathrm{y}_{\mathrm{i}}$, an exact filter is design as follow:

$$
\mathrm{H}_{\mathrm{i}}^{*}(\mathrm{w}, \mathrm{v})=\frac{\tilde{y}_{\mathrm{i}}(\mathrm{w}, \mathrm{v})}{\tilde{x}_{\mathrm{i}}(\mathrm{w}, \mathrm{v})}
$$

where the division is element by element between the FT of the output objective $\tilde{y}_{\mathrm{i}}$ and the FT of the training image $\tilde{x}_{\mathrm{i}}$. The ASEF filter is presented in equation (7) [7]:

$$
\mathrm{H}_{\mu}^{*}(\mathrm{w}, \mathrm{v})=\frac{1}{\mathrm{~N}} \sum_{\mathrm{i}=1}^{\mathrm{N}} \mathrm{H}_{\mathrm{i}}^{*}(\mathrm{w}, \mathrm{v})
$$

\section{Performances of the Correlation Filters in Face Recognition}

In computer simulations we considered a target set of 40 known subjects and a query set of 400 facial images of unknown subjects for identification. Each target subject set has 10 facial images for build a filter. The facial identification is performed by crosscorrelating a filter with each of the query set and processing the 400 correlation outputs on which only 10 facial images belongs to an authentic subject. Each one of the correlation output is searched for peaks and height of these peaks are used to determine whether the facial image is of an authentic or impostor subject.

The threshold for an authentic subject is setup with the smallest PSR value of the 10 correlation outputs of the authentic subject. Thus, performed a cross-correlating 40 filters with the query set, we obtain 16000 correlation outputs, of which only 400 are of authentic subjects. 
Some facial images that contain the target set and query set has distortions such as: facial expressions, small degrees of rotation, profile (left end right) and, partial occlusion caused by sunglasses, beard and mustache. Sample ORL training face images are shown in Fig. 2 [9]. Each facial image was cropped and scaled manually to a resolution of $64 \times 64$ pixels.

One of the main problems facing the facial processing systems is the variation in lighting [10]. To address this problem, it has been proposed methods that combine samples of facial images affected by illumination [11], while in [12] performed normalization in facial images. In this paper we used the logarithmic transformation to improve the intensity of the pixels in the shaded region and even with the light regions as described in [10]. Fig. 3 shows how the log transformation improves illumination in facial images.
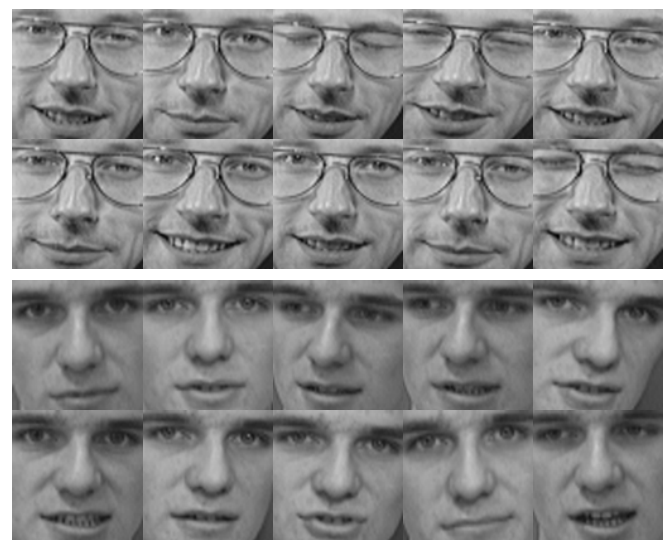

Fig. 2. Sample ORL data set. Training set for subject 27 (top) and subject 30 (bottom).
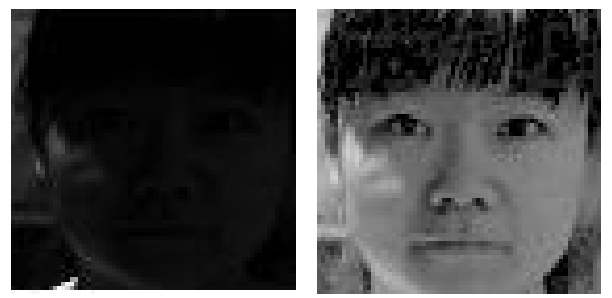

Fig. 3. Logarithmic transformation for improve the intensity of the pixels in shaded areas. Left: original image and right: image improved.

An advantage to establishing a PSR threshold for each authentic subject is that false rejection rate is low and the accuracy of algorithms based in correlation is significantly improved. Other advantage on this procedure is that in case that an algorithm receives as input a test facial image that improve the PSR threshold established for these subject, then is possible run an update to the filter over the time, getting a precise and exact recognition. Figs. 4 and 5 shows box plots for the best K-Law filter PSR performance (subject 27, showed in fig. 2) and the worst PSR performance (subject 1), respectively. 


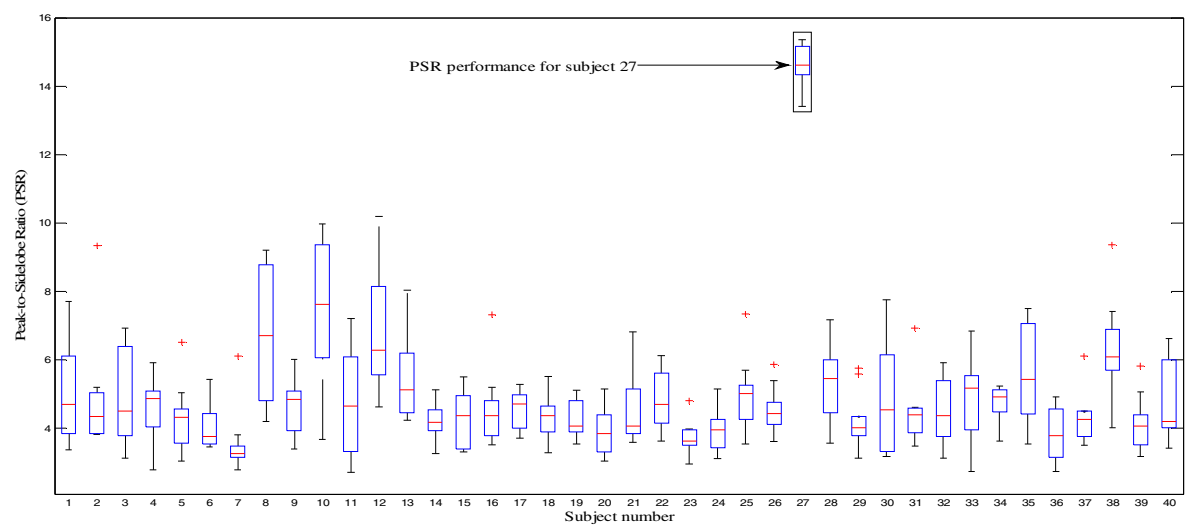

Fig. 4. Best K-Law filter PSR performance (subject 27)..

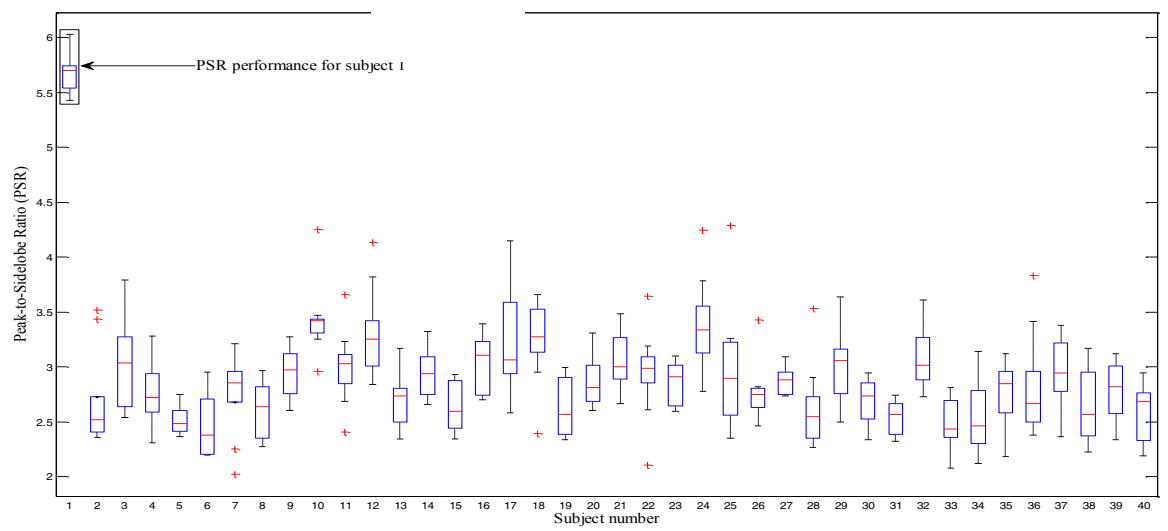

Fig. 5. Worst K-Law filter PSR performance (subject 1)

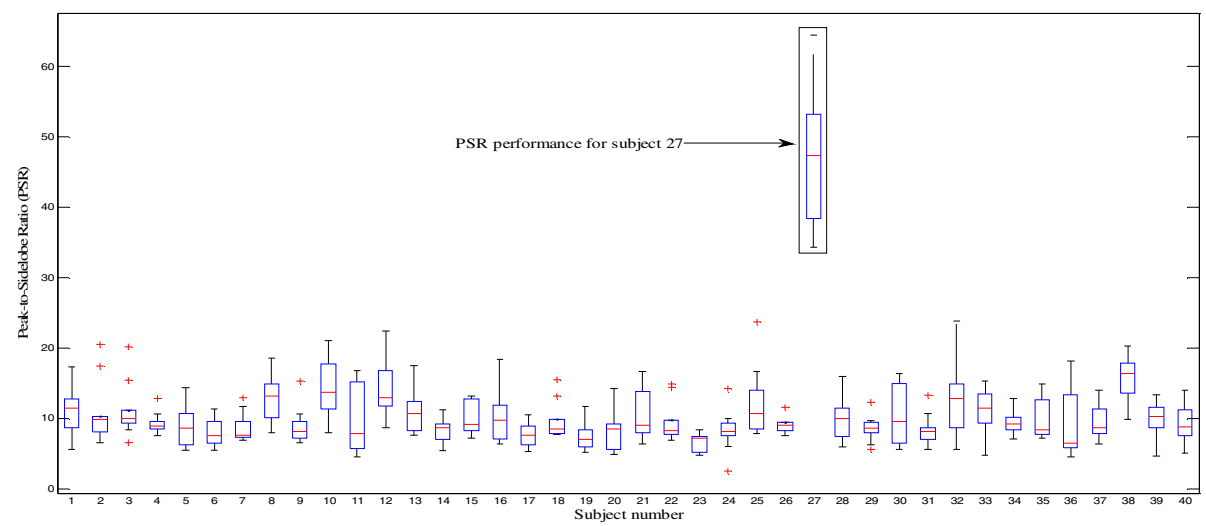

Fig. 6. Best MACE filter PSR performance (subject 27) 


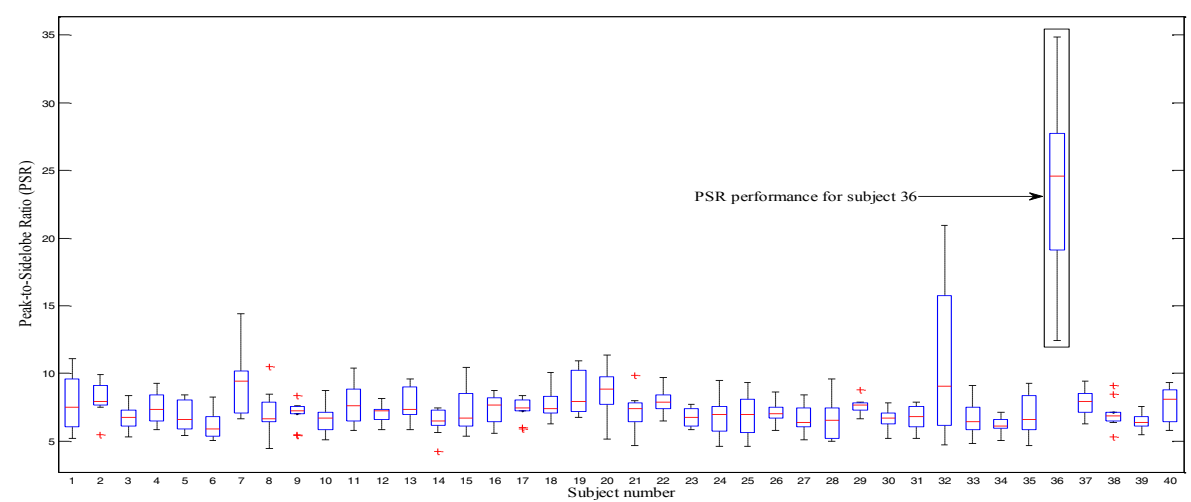

Fig. 7. Worst MACE filter PSR performance (subject 36)

Figs. 6 and 7 shows the best and worst MACE filter PSR performance. Although [13] obtained a recognition rate of $100 \%$ with the MACE filter, in these study only was considered facial images with facial expressions, no other distortions used as in this work.

ASEF was originally applied in eye localization, showing a good performance. This paper presents the results of this filter applied in facial recognition. The experiment showed that this filter produces PSR values greater than or equal to 14 for authentic subjects. Figs. 8 and 9 show the box plots for the best and the worst ASEF filter PSR performance, respectively. This filter shows a poor discriminating capacity, it produce PSR values greater that threshold for impostors subjects and, do not recognized correctly many facial images of authentic subjects.

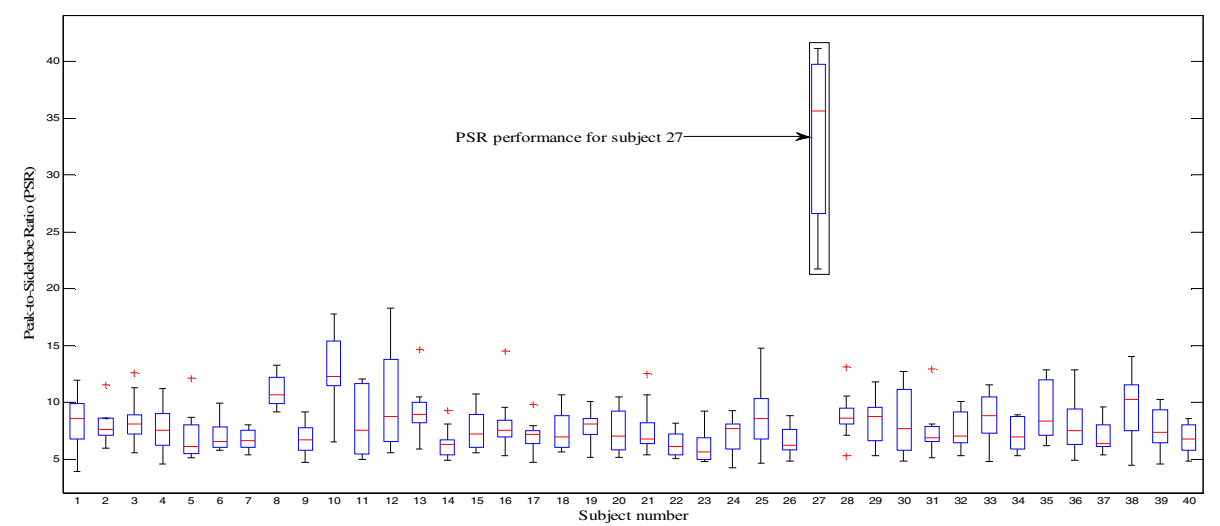

Fig. 8. Best ASEF filter PSR performance (subject 27) 


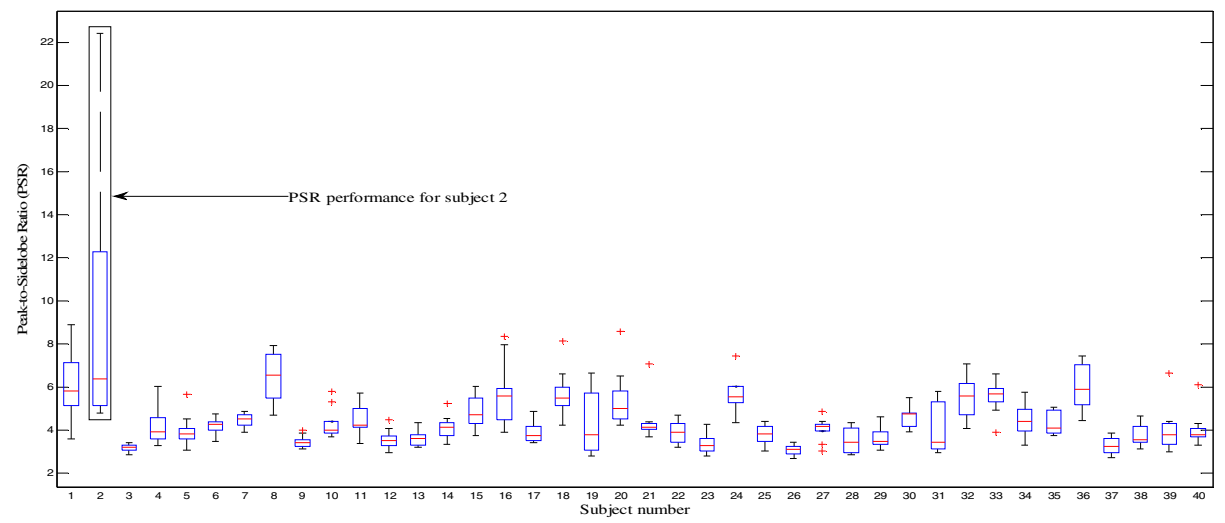

Fig. 9. Worst ASEF filter PSR performance (subject 2)

The performance of correlation filters in face recognition is summarized in Table 1. As can be see, the K-Law nonlinear composite filter with a nonlinear factor $\mathrm{k}=0.3$ offers the best performance with a recognition rate of $100 \%$. The filter K-Law states two different facial images of different subjects as equals the $0.2 \%$.

Where FAR (False Accept Rate) is the percentage of time that is reported as equal to two individuals who are not, FRR (False Rejection Rate) which is the percentage of times that two equal individuals are wrongly considered to be different and, RR (Recognition Rate) which is the percentage of times it correctly recognizes a subject and $\mathrm{AC}$ (Accuracy) is the proportion of the total number of predictions that were correct by the classifier.

Table 1. Performance of correlation filters in face recognition

\begin{tabular}{lllll}
\hline Correlation filter & FAR $\%$ & FRR $\%$ & RR\% & AC\% \\
\hline K-Law & 0.2 & 0 & 100 & 99.50 \\
MACE & 11.5 & 0 & 100 & 89.69 \\
ASEF & 316 & 0 & 100 & 24.04 \\
\hline
\end{tabular}

\section{Conclusions}

This document provides a brief assessment of the performance of correlation filters in facial recognition problem. The experiments show that K-Law nonlinear composite filter with logarithmic transformation to the facial images, both test and training achieved a recognition rate of $97.5 \%$. The algorithm that implemented this filter had a $99.50 \%$ of accuracy. It also shows that the quality of images affected from variations in lighting was improved by applying the logarithmic transformation. Advantages of the correlation method include shift-invariance and ability to suppress impostor faces using a PSR threshold. We are currently improving the filters design methods and testing the correlation filters on the much larger database, including different pose, illumination, expressions, scale and rotation variations. 
Acknowledgments. Credit is hereby given to the Massachusetts Institute of Technology and to the Center for Biological and Computational Learning for providing the ORL database. This work was financed by CONACYT through the scholarship provided by the first author (CONACYT 45360/344833). This work has been developed within the program Maestría y Doctorado en Ciencias e Ingeniería (MyDCI) at UABC.

\section{References}

1. National Science and Technology Council, http://biometrics.gov

2. Vijaya Kumar, B., Mahalanobis, H., Juday, R.: Correlation Pattern Recognition. Cambridge University Press, New York (2005)

3. Gonzalez-Fraga, J.A., Kober, V., Alvarez Borrego, J.: Adaptive Synthetic Discriminant Function Filters for Pattern Recognition. Optical Engineering 45, 057005 (2006)

4. VanderLugt, A.B.: Signal detection by complex spatial filtering. IEEE Transactions Information Theory 10, 139-145 (1964)

5. Casasent, D., Chang, W.: Correlation synthetic discriminant functions. Applied Optics 25, 2343-2350 (1986)

6. Javidi, B., Wang, W., Zhang, G.: Composite Fourier-plane nonlinear filter for distortioninvariant pattern recognition. Optical Engineering 36, 2690 (1997)

7. Bolme, D.S., Draper, B.A., Ross Beveridge, J.: Average of Synthetic Exact Filters. Computer Science Department Colorado State University, Fort Collins (2010)

8. Samaria, F., Harter, A.: Parameterization of a stochastic model for human face identification. In: 2nd IEEE Workshop on Applications of Computer Vision, Sarasota (1994)

9. Savvides, M., Vijaya Kumar, B.V.: Illumination normalization using logarithm transforms for face authentication. In: Kittler, J., Nixon, M.S. (eds.) AVBPA 2003. LNCS, vol. 2688, pp. 549-556. Springer, Heidelberg (2003)

10. Sim, T., Kanade, T.: Combining Models and Exemplars for Face Recognition: An Illuminating Example. In: Proceedings of the CVPR (2001)

11. Belhumeur, P., Hespanha, J., Kriegman, D.: Eigenfaces vs Fisherfaces: Recognition Using Class Specific Linear Projection. In: PAMI-19 (1997)

12. Savvides, M., Vijaya Kumar, B.V., Khosla, P.: Face Verification using Correlation Filters. In: Proc. of the third IEEE Automatic Identification Advanced Technologies, Tarrytown, NY, pp. 56-62 (2002) 